\title{
Anatomic Study of Endoscopic Transnasal Approach to Petrous Apex
}

\author{
HE WENYUAN ${ }^{1}$, QIN QINGYING ${ }^{1}$, WANG GUXIAN ${ }^{1, \mathrm{a}}$ \\ ${ }^{1}$ QUJING MEDICAL COLLEGE
}

\begin{abstract}
With the development of Neurosurgery technology, there has been a qualitative leap forward with the appearance of microanatomy, which makes the deep brain tumors which were hard to be achieved in the past, and effectively reduces the mortality of patients. Petrous apex is a cone-shaped part of the anteromedial part of the temporal bone, which is deep. It has been a challenging area for surgical anatomy for a long time. In this paper, fresh adult perfused cadaveric head specimens and dry adult cadaveric head specimens were taken as the experimental objects. The anterior wall of sphenoid sinus and the internal septum of sphenoid sinus were excised under neuroendoscope. The structures of the lateral wall of sphenoid sinus were identified and dissected. The lateral wall of sphenoid sinus and the bone of skull base were opened with micro drill, The meninges were exposed and cut open, and the related structures were dissected, observed and photographed. The experimental results show that it is relatively safe to operate in the range of less than $8 \mathrm{~mm}$, and the rock tip can be found accurately. The measurement of the bony structure of the skull base is helpful for the surgeon to judge the course of the internal carotid artery and its adjacent structure.
\end{abstract}

\section{Introduction}

Neuroendoscopy was first used to deal with sellar lesions. In recent years, with the improvement of endoscopic surgical instruments and techniques, neuroendoscopy has been paid more and more attention by neurosurgeons. With the continuous promotion of neuroendoscopic technology, the indications of neuroendoscopic surgery are expanding. Now it can be applied to deal with a variety of diseases and achieved good therapeutic effect.

Endoscopic transnasal approach is becoming a new research field in skull base surgery,. $G$ ü nter reviewed guidelines for reprocessing patients with suspected or confirmed prion disease after flexible endoscopy. G ü nter suggested that in case of delay of suspected prion disease, routine use of alkaline detergent can ensure disposable use of brush and detergent, and consider verified reprocessing as appropriate [2-3]. Li discussed the effect of sphenoid cartilage tympanoplasty under nasal endoscope in the treatment of anterior quadrant tympanoplasty. Li's experiment shows that endoscopic butterfly inlay tympanoplasty is a reliable and minimally invasive alternative to repair anterior tympanic membrane perforation, with high closure rate and low complication rate [4-5].Huang reviewed the current literature to determine the use of thrive in patients with apnea undergoing shared airway surgery[6-8]. A 28 year old patient with inferior canthus underwent epicanthoplasty via Y-V epicanthoplasty. Choi designed a simple Y-V epicanthoplasty incision, which provided enough surgical field to effectively locate the inner canthus tendon [9-10].
In recent years, with the development of nasal endoscopy, it is possible to deal with the lesions in the petrous apex region through the nasal approach, and it can reduce the damage relatively. In this paper, the anatomic characteristics of petrous apex region in general, endoscopy and microscope were discussed through the study of adult cadaveric head perfused with latex, which provided some anatomical reference for the operation of petrous apex and even intracranial lesions by nasal endoscopy.

\section{Anatomy and Endoscopy from the Nose to the Petrous Tip Area}

\subsection{Anatomy from Nasal to Petrous Apex}

Petrous bone is located at the junction of the middle and posterior cranial fossa, which is the bridge connecting the middle and posterior cranial fossa. Its front is connected with sphenoid bone, and its medial side is at the junction of occipital bone. It is rich in peripheral nerves and blood vessels, and is the prone site of intracranial tumors. Clinically, it can be divided into two types: epidural and epidural. Intradural tumors include meningioma, neurilemmoma, epidermoid cyst, dermoid cyst and cavernous hemangioma. Epidural tumors include: myeloma, chondroma, chondrosarcoma. Although most of the tumors in this region are benign tumors, because of their clinical symptoms are not obvious, the tumors often grow larger, and the location is deep, and the blood supply is rich. It is closely related to the brain stem and vertebral 
basilar artery system, and the surrounding structures are complex and important. The anterior petrosal approach is mainly used to remove the petroclival region and cerebellopontine angle area by grinding the petrous apex at the middle skull base and the petrous bone behind it. At present, it is mainly used to remove tumors communicating with the middle and posterior cranial fossa, small tumors in the petroclival region, tumors spreading more into the internal auditory canal in the cerebellopontine angle area, basilar artery aneurysms, anterior inferior cerebellar artery aneurysms, etc. Among them, the area surrounded by the lateral margin of trigeminal nerve, greater superficial petrosal nerve, arcuate eminence and petrosal crest is the main scope of the ablation. How to avoid the internal carotid artery, facial auditory nerve, abducent nerve, cochlea and inferior petrosal sinus is the core of anterior petrosal approach.

\subsection{Endoscopic Observation}

Endoscopy can flexibly change the angle in the nasal cavity and observe the surrounding anatomical structure through the natural human foramen. It can provide the operator with an unobstructed visual field and an operation channel without cerebral retraction, which greatly improves the quality of petrous apex surgery. The purpose of this study is to explore the anatomic characteristics of petrous apex through endoscopic exposure on cadaveric head specimens, so as to provide anatomical reference for clinical endoscopic surgery. Traditionally, most of the space that is difficult to observe by microscope can be clearly exposed through endoscopy or endoscopic assisted technology. Endoscopy is more and more used in the treatment of petrous apex lesions. The anatomic structure around the petrous apex is complex and closely related to the internal carotid artery, pterygoid tube and eustachian tube. The injury of the internal carotid artery in the endoscopic transnasal approach to the petrous apex is the most serious complication. Accurate intraoperative judgment of the internal carotid artery is a necessary condition for safe access to the petrous apex and to avoid internal carotid artery injury.

\section{Experimental Materials and Methods}

\subsection{Experimental Materials}

There were 10 adult cadaveric heads, including 10 male and 10 female cadaveric heads. All the specimens were from the Department of anatomy, otorhinolaryngology Research Institute, regardless of age. In order to better show the relationship between nerves and blood vessels, the arterial system of cadaver head was perfused with color latex, and the vein was not treated. The related structures were dissected and photographed under endoscope.

\subsection{Experimental Methods}

The selected specimens were well fixed, intact appearance, no obvious trauma, close to normal color, no decay and deterioration, intact large vessels in the neck, tough cervical medulla, light yellow or white color. The specimens were numbered according to the sequence of anatomy and measurement. The heads of 10 fresh adult perfused cadaveric heads were fixed according to the position of endoscopic surgery. Both sides of the nasal cavity simulated the surgical exposure from the nasal to the petrous apex. The sphenopalatine foramen could be found in the posterior margin of the stump of the middle turbinate, and the sphenopalatine nerve and sphenopalatine artery passed through it. The dense connective tissue was removed from the ruptured foramen. The eustachian tube was separated and pulled outward to expose the tissue in the front of the internal orifice of carotid artery.

\section{Anatomic Analysis of Endoscopic Transnasal Approach to Petrous Apex}

\subsection{Analysis of Sphenoid Sinus Stage}

Protrusions and recesses help us reach the apex safely under endoscope. In the stage of gross anatomy measurement, the data from the anterior nasal spine to these important protrusions and recesses were measured with the anterior nasal spine as the base point. The distance from anterior nasal spine to sphenoid sinus is shown in Table 1.

Table1. Distance from anterior nasal spine to related structures of sphenoid sinus

\begin{tabular}{cccc}
\hline Anatomy & Max & Minimum & $\begin{array}{c}\text { Mean deviation } \pm \\
\text { standard deviation }\end{array}$ \\
\hline Anterior Wall of Sphenoid Sinus & 61.4 & 41.26 & $51.92 \pm 0.47$ \\
Sphenoid junction & 67.21 & 51.36 & $56.49 \pm 0.28$ \\
Midpoint of saddle & 89.58 & 63.73 & $65.29 \pm 0.57$ \\
$\begin{array}{c}\text { Optic canal midpoint } \\
\text { Midpoint of anterior sella of }\end{array}$ & 70.41 & 56.93 & $61.73 \pm 0.46$ \\
$\begin{array}{c}\text { internal carotid artery } \\
\text { Midpoint of inferior sella of }\end{array}$ & 68.17 & 54.79 & $60.82 \pm 0.49$ \\
$\quad$ internal carotid artery & 74.26 & 56.52 & $62.16 \pm 0.52$
\end{tabular}


Midpoint of posterior sella of internal carotid artery
The development and gasification of sellar sphenoid sinus is good, which provides an important basis for identifying the structure of sphenoid sinus in simulated operation. After resection of the anterior wall of the sphenoid sinus, the sphenoid sinus septum in various directions can be found. The distance analysis between the anterior nasal spine and the related structures of sphenoid sinus is shown in Figure 1.

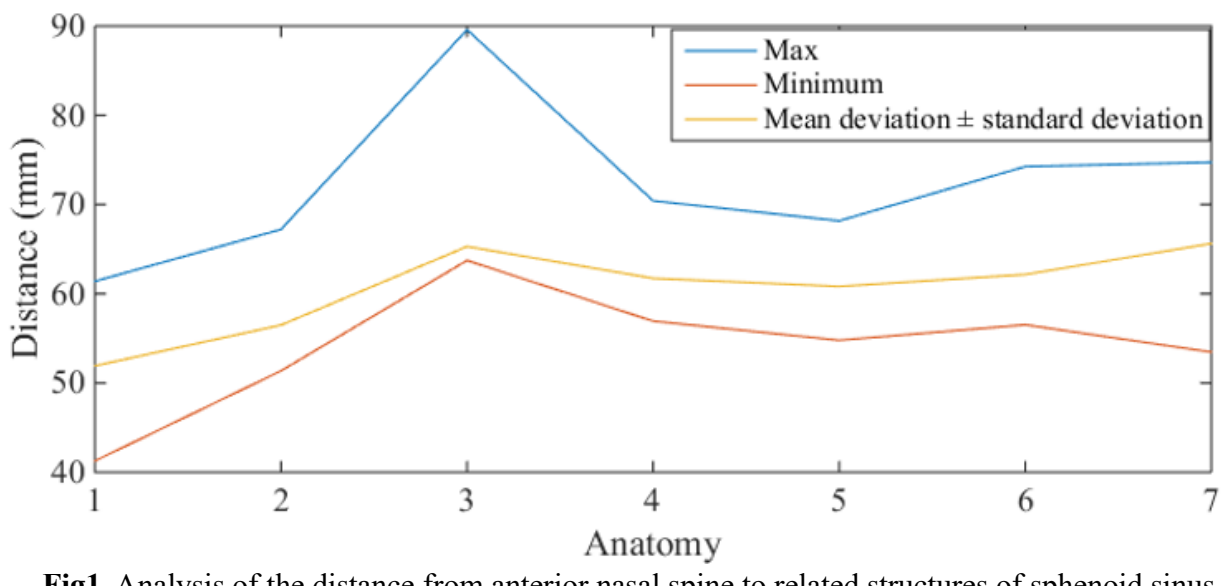

Fig1. Analysis of the distance from anterior nasal spine to related structures of sphenoid sinus

The maximum and minimum distance from anterior nasal spine to the midpoint of optic canal were $70.44 \mathrm{~mm}$ and $56.52 \mathrm{~mm}$, and the mean \pm standard deviation was $(61.73 \pm 0.46) \mathrm{mm}$. The internal carotid artery bulge was divided into anterior segment, inferior segment and posterior segment. The external upper boundary is the optic nerve canal, the inner and lower boundary is the parasellar carotid artery canal, the medial is the sella, and the lateral is the bone of the cavernous sinus. In order to better match the structure of the lateral wall of sphenoid sinus with the intracranial structure, and to provide a reference for clinical approach, this paper also used a pin to insert from the lateral structure of sphenoid sinus to the lateral side, and penetrated through the intracranial structure. It is found that the optic nerve internal carotid artery recess corresponds to the intracranial optic nerve Keyring anterior clinoid recess. Draw a line between the midpoint of the anterior segment of the unilateral internal carotid artery protrusion and the center of the clivus recess, take the midpoint of the line, and then make a circle with the radius less than $8 \mathrm{~mm}$. It is relatively safe to operate within this range, and the petrous apex can be accurately found.

\subsection{Measurement and Analysis of Important Structures in Lateral Skull base of Petrous Apex}

The distance from the inner edge of foramen ovale, foramen spinosum, foramen rupture, the orifice of pharyngeal tympanic duct, the inner edge of anterior and posterior border of carotid artery tube to the median line were measured. The results of statistical analysis showed that there was no statistical difference between the left and right sides. The distance between the adjacent structures of the petrous apex and the anterior nasal spine is shown in Figure 2.

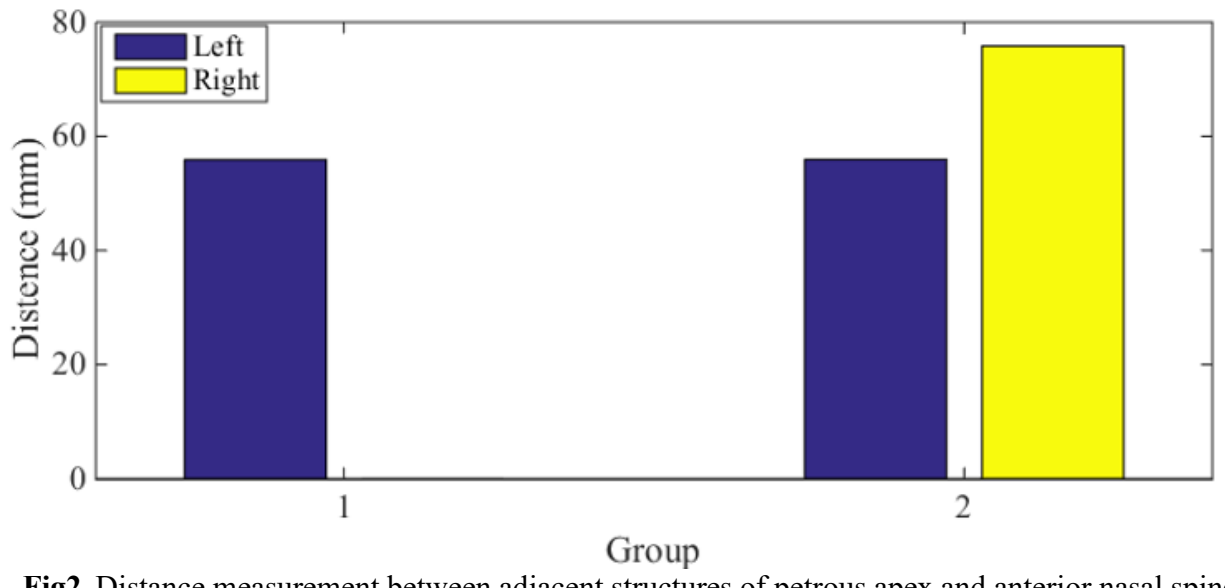

After statistical analysis, the results showed that there was statistical difference between male and female, and female measurement data was smaller than male; the distance from anterior nasal spine to anterior border of rupture foramen and anterior boundary of carotid artery tube was measured respectively. After statistical analysis 
of left and right data, there was no statistical difference between left and right sides. The measurement of the bony structure of the skull base is helpful for the surgeon to judge the course of the internal carotid artery and its adjacent structure.

\section{Conclusions}

When entering the endoscope, it should not exceed the posterior edge of the vertical segment of internal carotid artery petrous part forward, and the maximum value should not exceed $13 \mathrm{~mm}$ posteriorly and outwards until the dura mater is exposed, and the lower limit should not exceed the upper edge of the horizontal segment of the petrous temporal bone. Such a bone window can meet the requirements of surgical field of vision and will not cause damage to important blood vessels and nerves. Transnasal approach has the advantage of providing better exposure and a direct view of the ventral side of the midline structure. If the operation is performed from inside to outside through the nerve bundles during the process of reaching the lateral cranial nerve lesion, the nasal approach may face the same risk of cranial nerve injury. The choice of approach should be based on the location, range and nature of the lesion. There are still some deficiencies in this paper. The sample selected in this paper is not large enough to obtain and verify some achievements made by predecessors. In this paper, the specimens of sphenoid sinus with sellar type were screened out by preoperative CT scanning, but the specimens of pre sellar type and thyroidium type were not studied.

\section{References}

1. Kirin I G . A Two-Channel Optical Circuit for the Delivery of Incoherent Radiation into the Illumination Channel of a Medical Endoscope[J]. Biomedical Engineering, 2019, 53(4):280-283.

2. Günter Kampf, Jung M, Suchomel M, et al. Prion disease and recommended procedures for flexible endoscope reprocessing - review of policies worldwide and proposal for a simplified approach[J]. Journal of Hospital Infection, 2020, 104(1):92-110.

3. Shigemori, Toshiaki, Orihara, et al. Capsule endoscope and capsule endoscope system[J]. Minimally Invasive Therapy \& Allied Technologies Mitat Official Journal of the Society for Minimally Invasive Therapy, 2018, 12(5):227-34.

4. Li Y, Liu H Q, Sheng Y, et al. Clinical study of butterfly cartilage myringoplasty for anterior quadrant tympanic perforation under endoscope[J]. Zhonghua er bi yan hou tou jing wai ke za zhi = Chinese journal of otorhinolaryngology head and neck surgery, 2020, 55(6):611-614.

5. Endo T, Tominaga $\mathrm{T}$. Use of an endoscope for spinal intradural pathology[J]. Journal of Spine Surgery, 2020, 6(2):495-501.

6. Huang L, Dharmawardana N, Badenoch A, et al. A review of the use of transnasal humidified rapid insufflation ventilatory exchange for patients undergoing surgery in the shared airway setting[J]. Journal of Anesthesia, 2020, 34(1):134-143.

7. Li J, Zhao M, Hadeer M, et al. Dose Response to Transnasal Pulmonary Administration of Bronchodilator Aerosols via Nasal High-Flow Therapy in Adults with Stable Chronic Obstructive Pulmonary Disease and Asthma[J]. Respiration, 2019, 98(5):1-9.

8. Choi B G , Kim Y H . Oblique transnasal wiring canthopexy via Y-V epicanthoplasty for telecanthus correction in a patient with Waardenburg syndrome[J]. Archives of Craniofacial Surgery, 2019, 20(5):329331.

9. Johnstone L, Tassone P, Burrows S, et al. Extended role of transnasal oesophagoscopy: a review of the literature $[\mathrm{J}]$. The Journal of Laryngology \& Otology, 2020, 134(6):1-6.

10. A H F , A S R V , Thomas A. Lutz $\S \|$, et al. Evaluation of Acute Mountain Sickness by Unsedated Transnasal Esophagogastroduodenoscopy at High Altitude[J]. Clinical Gastroenterology and Hepatology, 2020, 18(10):2218-2225. 